through a scribbler of up to three swifts, and with one or more workers and strippers per swift. The device can also be used for predicting the effect of automatic controllers on the behaviour of the card, particularly in respect of possible hunting of the proposed control. This application of electronic computer techniques must mean the saving of much valuable time, to say nothing of the large amounts of actual raw material which would otherwise have to be processed to obtain the required results.

Fibre movement in roller drafting has been studied with the aid of radioactive tracers, the principle being to mix at random into a sliver a certain proportion of labelled fibres, and then to follow the movement of these fibres in the body of the sliver as it passes through successive drafting operations by standard procedures.

In the biological field, work on the identification of fibres by use of the sequence in cuticular scale pattern along the lengths of the fibres has led to a new classification of scale patterns for general use and has given more sensitive criteria for the identification of fibres. Such work may well have valuable applications outside the textile field-for example, in forensic science.

While the above examples may perhaps be cited as outstanding features of the Association's research programme, they are accompanied by a very great deal of less spectacular, but nevertheless invaluable, work of a routine nature.

The problem remains, and is recognized as fundamental by all workers in technological research, of getting the results obtained in the laboratory translated as rapidly as possible into practice in industry. The textile industry is probably no more backward in this respect than others; but it certainly remains true that much information which could, if thoroughly applied, make a very great difference to the technical efficiency of the industry, remains literally a closed book so far as the latter is concerned. This is not necessarily due to any strong prejudice against scientific research, but rather to the difficulty of securing adequate liaison between laboratory and the mill. The Wool Industries Research Association has made a notable attempt to surmount this difficulty by the appointment of liaison officers for this sole purpose. To quote the report on this matter : "The officers' visits give contributory members the advantage of verbal amplification of the Association's publications, and the advantage of discussing the firm's particular problems on the spot. At the same time these discussions enable the officers to keep the Association's staff informed of contemporary conditions and developments in the industry; thus research work can be directed along the most beneficial lines. Points of interest to members are discussed which often result in an investigation being pursued, to the members' very considerable advantage. The officers are also able to arrange for the co-operation of member firms in experimental projects which require large-scale trials or surveys. This two-way passage of information and of the use of equipment was scarcely anticipated when the liaison officers were appointed, but there is no doubt that it is now a valuable feature of their activities".

Such schemes of interchange are, of course, by no means novel in the industry, and are shared by other research associations and by university departments; but it is heartening for those whose foremost aim is the technical betterment of industry through sound research to see the process enlarged.

\section{THE FRAMEWORK OF ASTRONOMY}

FoR his inaugural lecture as Perren professor of $f$ astronomy in the University of London, which he gave last year at University College, London, Prof. C. W. Allen chose as his subject "The Framework of Astronomy". At the beginning of this lecture, which has now been published*, Prof. Allen points out that the work of the astronomer usually falls into one or the other of two categories: the expanding category, in which are the activities of elaborating, detailing, compiling and investigating individual objects for their own sake; and the contracting category, which includes the work of unifying, consolidating and standardizing. Very appropriate analogues are found in the political realm-namely, individualism and freedom in the first category, and regimentation and good order in the second. Both approaches are necessary in astronomy. The expanding individualism leads to new ideas and discoveries and also to long observational programmes with their risk not only of drudgery but also of the absence of discovery; equally important, however, is the regimentation mentioned under the contracting category, in which the co-ordination of the original facts and even, if required, the addition of missing links, tend to strengthen the subject. Such coordinated results frequently reveal not only regularities but also departures from anticipated laws, and the work of consolidation is responsible for results which can be used as a basis for further advances. The interconnexion of consolidated results from different branches of astronomy should lead to a strong stable framework of astronomical knowledge on which the future can build, and a great part of Prof. Allen's lecture is devoted to a consideration of the relation between the framework and the subject as a whole and also of the problems of constructing and using this framework. For the purpose of the lecture the following definition of 'framework' is given : "It is a complete assemblage of interconnected basis facts and quantities of astronomy prepared in readiness for use". It is admitted that no such framework exists; but as the possibility of its existence is not excluded, Prof. Allen discusses it under the above form.

An efficient framework for astronomy would contain information that represents it as a whole rather than the results of particular individuals and would be "concise, factual, and quantitative". An example is taken from the case of a comet. As every computer of cometary orbits knows, from at least three observations over about a week an orbit is computed on the assumption of parabolic motion and from this an approximate ephemeris enables observers to find it for a few weeks. Subsequent observations provide the data for a more accurate orbit, including the eccentricity, to be computed; then finality can be obtained when all the observations (probably extending over several months) are published and, taking planetary perturbations into consideration, a definitive orbit is computed. No improvement is possible with existing observations; but on the comet's next return the procedure could be repeated, and the sets of elements obtained, which represent the definitive orbits, then approach the ideal for the

* The Framework of Astronomy : an Inaugural Lecture delivered at University College, London, 1 May 1952. By C. W. Allen, Perren Professor of Astronomy in the University of London. Pp. 17. (Published for the College by $\mathrm{H}$. K. Lewis and Co., Ltd., 1952.) 38 . 
quantitative framework, that is, "it is efficient, representative, accurate and stable". This is typical of most branches of astronomy where there is a hope that improved values will become available and later work will reap the benefit, but there is also the fear that an improvement will change the adopted values and upset work in progress: The analogue of the definitive orbit cannot, however, be used in other branches of astronomy, because values never reach real stability, and in consequence astronomers may set up either a 'rigid' or a 'flexible' system.

In the rigid framework values of all the important constants, as close as possible to the best determinations at the time of adoption, are selected; but if these values are later found to be in error they are not changed, a correction being applied for those cases that require it, and such a correction can absorb all the effects of improvements in the experimental values. While a rigid framework of this type is necessary for accurate positional astronomy, the one in use at present does not attain perfection ; but the data of its framework are used for the preparation of predictions, and, on comparing these with observational results, discrepancies frequently reflect an inaccuracy in the framework data. Whether such inaccuracies occur or not, the original data remain unaltered so that the process of predicting and observing can continue without interruption, and a clear interpretation can be placed on the observations. This rigid system is not, however, suitable in all cases, and in the more rapidly growing subject of astrophysics, where accuracy is relatively low, a flexible system that caters for perpetual improvement is more appropriate. Here, where the quantitative values vary, the progressive astronomer adjusts his outlook to the knowledge that the values are not precisely known, and he is pleased when the constants fluctuate within the domain of their probable errors. This flexible system shows hope of being available for the whole of astronomy, including astrophysics.

The themo is developed by Prof. Allen; but it is impossible in the limited space to deal with the numerous applications referred to, and this article can do little more than direct attention to the importance of the lecture. Towards the end three conditions, as follows, are mentioned as necessary for building an item into the framework of astronomy : a clear concept and suitable definition of the item; evidence that the item is vital to the progress of astronomy; and some estimate of its numerical value. The mean density of the universe is taken as an example on the above basis, although its value is not accurately known. While the density of the visible part made up of stars in external nebulæ is in the neighbourhood of $10^{-4} \mathrm{gm} . / \mathrm{cm} .^{3}$, this neglects the possibility of tenuous matter in the inter-nebulæ spaces. For various reasons, including the meagre observational evidence relating to this space, it is pointed out that, if we accept the extreme view of the reddening of the nebulæ as due entirely to gas, the estimate of the mean density of the universe would be increased by a factor of about ten thousand. When the mean density of the universe is open to such doubt, it is realized how far astronomy has still to go. In spite of this doubt, however, the density of the universe has its place in the development of cosmology, and, if it can be placed between the limits $10^{-26} \ldots 10^{-30} \mathrm{gm} . / \mathrm{cm} .^{3}$, it may possibly be restricted to a narrower range in a few years. "It is already built into the astronomy framework as a quantity to be watched and improved by all means available. If we had no other use for astronomy framework, it would at least be interesting and instructive to see it change as the years go by."

\section{COMPOUNDS OF TERTIARY AMINES WITH NITROSYL CHLORIDE AND WITH DINITROGEN TETROXIDE}

\section{By DR. ALAN E. COMYNS}

University College, Gower Street, London, W.C.I

$A^{\mathrm{L}}$

LIPHA'TIC and aromatic tertiary amines have been found to react in inert solvents at $-78^{\circ}$ with nitrosyl chloride and with dinitrogen tetroxide to yield coloured complexes. The aliphatic complexes are brown and the aromatic plum-coloured. They are insoluble in hydrocarbons but soluble in chlorinated hydrocarbons. The formation of such a complex by trimethylamine and nitrosyl chloride was observed by Jones and Whalen ${ }^{1}$, but the generality of this reaction, and its significance in connexion with the reactions of amines with nitrosating agents, have not hitherto been recognized. Amines exhibiting this phenomenon include : trimethylamine, triethylamine, dimethylaniline, diethylaniline, $p$-nitroso-dimethylaniline and $\mathrm{N}$-methyldiphenylamine. The three nitro-dimethylanilines did not form such complexes.

As the complexes are stable only at low temperatures, conventional methods of analysis are inapplicable; the molecular ratios in which the reactants had combined in two of the complexes were determined as follows. Known quantities of the two reactants were mixed at low temperature in pentane solution, and that in excess was quantitatively removed by pumping under high vacuum into a trap cooled in liquid air, the reaction vessel being maintained at $-78^{\circ}$ or $-100^{\circ}$. The excess, collected in the liquid air trap, was then determined. The complexes studied by this means were those of dimethylaniline with nitrosyl chloride in excess, and of dinitrogen tetroxide with trimethylamine in excess. The results are shown in the accompanying tab'es.

These results establish the formation of $\left({ }^{\circ} \mathrm{H}_{5}\right)$ $\left(\mathrm{CH}_{3}\right)_{2} \mathrm{~N} . \mathrm{NOCl}$ at $-78^{\circ}$, and of $\left(\mathrm{CH}_{3}\right)_{3} \mathrm{~N} \cdot \mathrm{N}_{2} \mathrm{O}_{4}$ at $-100^{\circ}$.

A good solvent for such complexes is a mixture of sym.-tetrachlorethane with ether (70:30 by volume). Qualitative observations of the absorption spectra of some of the complexes were made at $-78^{\circ}$ in this solvent. Solutions containing an excess of dimethylaniline with nitrosyl chloride and with dinitrogen tetroxide had almost identical absorption maxima. That with nitrosyl chloride appeared at $398 \mathrm{~m} \mu$; and that with dinitrogen tetroxide at $404 \mathrm{m \mu}$.

The electrical conductance of dimethylaniline with nitrosyl chloride was examined in the same solvent

Table 1. Dimterylaniline with NItrosyl ChLORIDR
Temp. $=-78^{\circ}$ $($ DMA $=$ dimethylaniline $)$

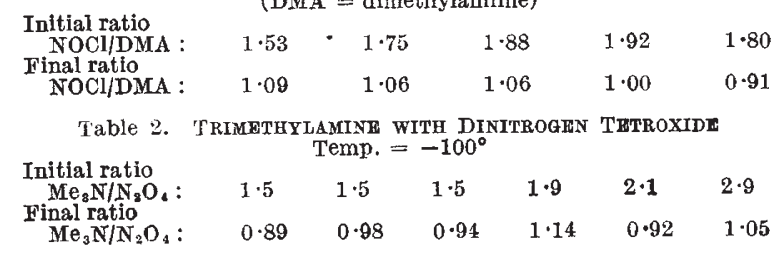

